\title{
An Energy-saving Model-based Air Balancing Method for the Ventilation System
}

\author{
Gang Jing ${ }^{1}$, Wenjiang $\mathrm{Cai}^{2}$, Cui Can ${ }^{2}$ \\ ${ }^{1}$ School of Tansportation and Logistics Engineering, Shandong Jiaotong University \\ Jinan 250023, P.R. China \\ venturejg@qq.com; ewjcai@ntu.edu.sg \\ ${ }^{2}$ School of Electrical and Electronic Engineering, Nanyang Technological University \\ Singapore 639798 \\ CCUI003@e.ntu.edu.sg
}

\begin{abstract}
In this paper, an energy-saving oriented model-based air balancing method aiming at reducing the energy consumption and achieving the air balancing among all air terminals is presented. The method includes two layers: one is the static pressure control layer, and the other is the air balancing layer. The first layer is to modulate the speed of the fan with the variance of total airflow rates to achieve the aim of energy saving. The second layer is to implement the air balancing among the terminals. Experimental tests are carried out in the experimental rig with five terminals to validate the performance of the proposed method.
\end{abstract}

Keywords: Ventilation system, Air balancing, Bayesian regression, Model-based method, Parameter identi-fication, Machine learning.

\section{Introduction}

Buildings account for $35-40 \%$ of total energy use in many countries $[1,2]$. For instance, in the USA, $41 \%$ of the energy is used in the buildings. In schools and hospitals, the need for energy for building services is audited to be just above $50 \%$ [3]. Therefore, it is motivated to investigate the energy saving potential in the building. The mechanical ventilation system is one of the most energy-demanding systems in the buildings, which operates on a 12-months basis.

Many methods, which focus on energy efficient ventilation systems, have been studied in the past decades, which can be classified into two categories: 1) testing, adjusting and balancing (TAB) method; 2) demand controlled ventilation (DCV) strategies. TAB is the three major steps used to achieve proper operation of heating, ventilation, and air conditioning (HVAC ) systems. The balancing is usually based on the design flow values required by the Mechanical Engineer for the project [4]. The $\mathrm{TAB}$ of the ventilation system is a time-consuming and laborious task that need iteration loops to ensure that the designed airflow rate is reached in every location of the building. Some studies have proposed the non-iterative TAB method, to reduce the time consumed. Federico Pedranzini et al. [5] presented the progressive flow (PFM) method, a non-iterative approach, by adjusting dampers progressively from the furthest terminal to the closets terminals. A control loop, which using the addon device, is used to decouple the interactive effect in the terminals. The number of measurements and the time consumed on air balancing are largely reduced, and only 57\%-67\% fewer adjustments are required compared to use the conventional ratio method. Tamminen et al. [6] presented another non-iterative TAB approach suited for small-scale ventilation systems with short common pipes, only needed the knowledge of the fan total airflow rate and pressure. During the adjustment process, the fan static pressure remains constant implemented by a variable speed drive fan with corresponding fan speed controller, while the total flow rate is increased until the estimated airflow rate meets design requirements. Simulation and experimental results validate this approach.

DCV system is the ventilation system with feed-back and/or feed-forward control of the airflow rate according to measured demand indicators which include airflow rate, pressure, temperature, humidity, $\mathrm{CO} 2$ or other gas concentration, particle concentration and occupancy. Englander et al. [7] proposed two control strategies utilizing terminal box DDC feedback to directly control the supply fan speed. All of the terminal box controllers were polled and an error signal was calculated based on the maximum or average difference between the setpoint and measured airflows. The energy saving potential of the two control strategies was verified using the simulation. Samira Rahnama et al. [8] proposed a method based 
on measuring the static pressure at the terminal dampers. The proposed DCV control was applied on a mockup of a VAV ventilation system, including a supply duct with four branches connected to four terminal dampers, in a laboratory environment. The experimental results showed a minimum reduction of $21 \%$ in main fan power use with the DCV control compared to constant static pressure.

Due to the time-consuming and laborious characteristics, the TAB method is not easily executed when the system needs to be rebalanced because of system aging and changing room usage. DCV strategies can regulate the airflow rate to each branch to acceptable indoor air quality while consuming the least energy. However, many studies [9] pointed out that DCV control strategy cannot adequately consider the ventilation demand in a space in many situations since the indicator (such as $\mathrm{CO} 2$, temperature) provides no information of other contaminants, such as those generated by building materials. What's more important is that the indicator is usually captured from a single critical zone, which will make the other zones overventilated or under-ventilated, resulting in unbalancing of the system and wasting of energy.

Proper air balancing can reduce the total airflow rate, while at the same time avoiding the over-ventilated or underventilated problem. Therefore, the air balancing method can save energy consumed by the ventilation system. In addition, the advantage of energy-saving of air balancing methods can reduce the amount of pollution created on-site of at the electricity generating plant, and also reduce the noise of the ventilation system. Recently, some methods focusing on the air balancing of ventilation system have been proposed $[10,11]$. These methods only realize the balance of the system using the machine learning or optimization algorithm, while not consider the energy saving. Therefore, a DCV system will have large potential of energy-saving provided that the DCV system is balanced compared to normal DCV system.

In this paper, an energy-saving model-based air balancing method which can accurately balance the DCV system is proposed. The method includes two schemes. One is the static pressure control scheme used for maintaining the static pressure of the main duct to its set-point. The other one is air balancing scheme used to balance the ventilation system by three steps: 1) building a mathematical model for duct system based on steady-state pressure balance; 2) identifying the significant model parameters by Bayesian regression supervised machine learning; 3) determining the damper position based on the formulated model. The first scheme is used to realize the energy saving and the second scheme is used to realize the balancing among the terminals. The proposed method is validated in an experimental testbed with five terminals.

\section{Energy-Saving Model-Based Air Balancing Method}

The proposed method consists of two layers, namely static pressure control layer and air balancing layer. The structure of the proposed method is illustrated in Figure 1.

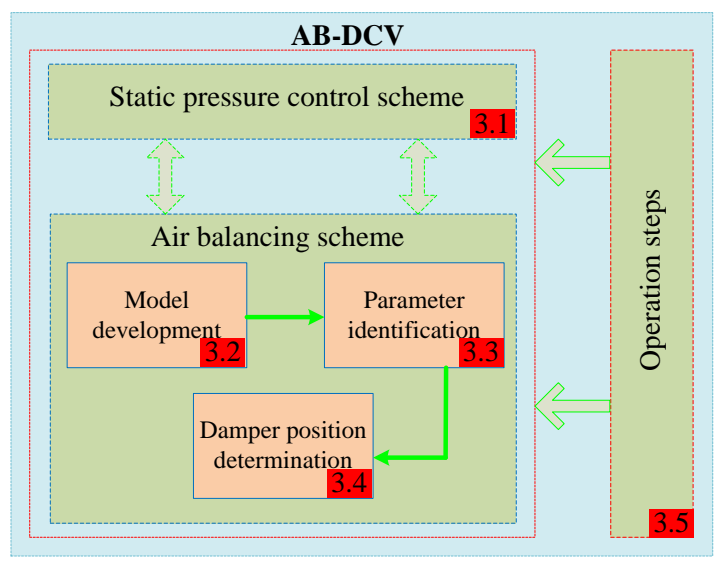

Fig. 1: Block diagram of the proposed method.

\subsection{Selection of the static pressure control layer}

The most common method of supply fan control in VAV systems is closed loop proportional-with-integral (PI) control utilizing the pressure measured in the main supply duct in which variable speed drives (VSDs) are commonly used due to their low energy consumption.

In this study, the closed loop PI control strategy is adopted for its simplicity and has been broadly used in practice. The PI controller used in this study can be mathematically expressed as: 


$$
U=K_{p} E+K_{i} \int E d T+U_{0}
$$

where $U$ is the output direct current voltage (in the experimental rig, the fan is a variable-speed centrifugal supply fan whose speed can be adjusted by direct current voltage over the range $[0-10 \mathrm{~V}]), E$ is the error/deviation of static pressure of the main duct, $K_{p}$ is the proportionality (gain), $K_{i}$ is the integral parameter, and $d T$ is the time change, $U_{0}$ is the output value in the absence of an error (i.e. $E=0$ ).

\subsection{Model development for the proposed method}

Straight duct, elbow, damper junction and transition are the elementary components in a ventilation system. The pressure-flow relationship of these components is complicated and difficult to formulate analytically. Fortunately, these fittings have been widely tested experimentally, and empirical formulas have been established based on the experimental results [12]. The model of the component is presented in the following equations.

\section{- Duct}

$$
\begin{aligned}
& \Delta P_{\text {duct }}=L \varphi_{\text {duct }}(q) \\
& \varphi_{\text {duct }}(q)=2000 f \frac{\rho q^{2}}{\pi^{2} D^{5}}
\end{aligned}
$$

where $\Delta P_{\text {duct }}$ is the friction loss in terms of total pressure, $f$ is the friction factor, $L$ is the duct length, $D$ is the duct diameter, $V$ is the air velocity, $\rho$ is the air density, $\varepsilon$ is the duct surface roughness, $R e$ is the Reynolds number, $v$ is the kinematic viscosity.

\section{- Elbow, damper, junction and transition}

$$
\begin{aligned}
\varphi(q) & =\Delta \mathrm{P} \\
& =\frac{1}{2} C_{f} \rho\left(\frac{4 q}{\pi D^{2}}\right)^{2}
\end{aligned}
$$

where $C_{f}$ is the friction coefficient of the elbow, damper, junction, and transition respectively. $\varphi_{\text {duct }}(q)$ and $\varphi(q)$ are the basis function of each component to be used in Bayesian Linear regression machine learning.

For modeling a duct system, an associate matrix $A$ is defined to describe the network topology. The elements $a_{i j}$ indicating the linkage between path $i$ and branch $j$ is defined as follow:

$$
a_{i j}= \begin{cases}0, & \text { branch } j \text { does not belong to the path } i ; \\ 1, & \text { branch } j \text { is a duct belonging to the path } i ; \\ 2, & \text { branch } j \text { is an elbow belonging to the path } i ; \\ 3, & \text { branch } j \text { is a junction belonging to the path } i ; \\ 4, & \text { branch } j \text { is a damper belonging to the path } i ; \\ 5, & \text { branch } j \text { is a transition belonging to the path } i ;\end{cases}
$$
follows:

A basis function taking linear combinations of a fixed set of component models (nonlinear functions) is defined as 


$$
\varphi\left(a_{i j}\right)= \begin{cases}0, & a_{i j}=0 \\ \varphi_{\text {duct }}(q), & a_{i j}=1 \\ \varphi_{\text {elbow }}(q), & a_{i j}=2 \\ \varphi_{\text {damper }}(q), & a_{i j}=3 \\ \varphi_{\text {junction }}(q), & a_{i j}=4 \\ \varphi_{\text {transition }}(q), & a_{i j}=5\end{cases}
$$

By the definition of associate matrix and basis function, the steady-state pressure balance gives:

$$
\sum_{j=1}^{N} \omega_{j} \varphi\left(a_{i j}\right)+P_{s p}=0
$$

where $\omega_{j}$ is the unknown variables of the component in the duct system, $P_{s p}$ is the static pressure set-point. Assuming a duct system has $M$ paths and $N$ branches, then $i \in(1, \cdots, M), j \in(1, \cdots, N)$.

Given one desired airflow rate of a terminal, the damper pressure difference of this terminal will be obtained:

$$
P=\sum_{\substack{j=1 \\ j \neq k}}^{N} \omega_{j} \varphi\left(a_{i j}\right)+P_{s p}
$$

where $k$ is the number of the damper branch, i.e. the damper model is not included in Eq.(7).

The associate matrix $A$ gives the detail description of the network topology, Eq.(6) provides the model of the duct network, and Eq.(7) provides the model of achieving the pressure of the damper.

\subsection{Model development for the proposed method}

In this section, the purpose is to identify the unknown parameters of the developed model of the duct system. The supervised machine learning method of Bayesian linear regression $[13,14]$ is employed to estimate the unknown parameters of the system.

Assuming the target pressure difference of the damper, $\mathrm{P}$, is given by a deterministic function $p(q, w)$ with additive Gaussian noise so that

$$
P=p(q, w)+\varepsilon
$$

where $\varepsilon$ is a zero mean Gaussian random variable with precision (inverse variance) $\beta$.

According to definition of the marginal distribution, conditional distribution, and Bayes' theorem for Gaussian variables, the posterior distribution can be obtained:

$$
p(\mathbf{w} \mid \mathbf{P})=N\left(\mathbf{w} \mid m_{N}, S_{N}\right)
$$

where

$$
\begin{aligned}
m_{N} & =\beta \mathbf{S}_{N} \boldsymbol{\Phi}^{T} \mathbf{P} \\
\mathbf{S}_{N}^{-1} & =\alpha \mathrm{I}+\beta \boldsymbol{\Phi}^{T} \boldsymbol{\Phi} \\
\boldsymbol{\Phi} & =\left(\begin{array}{llll}
\varphi_{0}\left(q_{1}\right) & \varphi_{1}\left(q_{1}\right) & \cdots & \varphi_{M-1}\left(q_{1}\right) \\
\varphi_{0}\left(q_{2}\right) & \varphi_{1}\left(q_{2}\right) & \cdots & \varphi_{M-1}\left(q_{2}\right) \\
\vdots & \vdots & \ddots & \vdots \\
\varphi_{0}\left(q_{N}\right) & \varphi_{1}\left(q_{N}\right) & \cdots & \varphi_{M-1}\left(q_{N}\right)
\end{array}\right)
\end{aligned}
$$

The optimal prediction of the pressure difference of the damper, for a new value of $q$, will be given by the conditional mean of the target variable. 


$$
\begin{aligned}
P & =\mathrm{E}[p(P \mid \mathbf{P}, \alpha, \beta)] \\
& =\int P p(\mathbf{P} \mid \mathbf{w}, \beta) p(\mathbf{w} \mid \mathbf{P}, \alpha, \beta) d P d \mathbf{w}
\end{aligned}
$$

\subsection{Damper position determination for the proposed method}

In this stage, the purpose is to determine the damper position according to the predicted pressure difference using Eq.(10).

Assuming a desired airflow rate $q$, the pressure difference $\mathrm{P}$ of the damper can be obtained according to Eq.(10). The damper position will be determined through the lookup table based on the obtained $C_{f}$. The friction coefficient of the butterfly damper in round conduit is tabulated with the damper position is shown in Table 1, which comes from the handbook of hydraulic resistance [22].

Table 1: Friction coefficient table for circular butterfly damper CD9-1.

\begin{tabular}{ll}
\hline Degree $(\theta)$ & Coefficient $\left(C_{f}\right)$ \\
\hline 0 & 0.19 \\
10 & 0.67 \\
20 & 1.76 \\
30 & 4.38 \\
40 & 11.2 \\
50 & 32.0 \\
60 & 113 \\
70 & 619 \\
75 & $2.01 \times 10^{3}$ \\
80 & $1.035 \times 10^{4}$ \\
85 & $9.9999 \times 10^{3}$ \\
90 & $9.9999 \times 10^{3}$ \\
\hline
\end{tabular}

The procedure of damper position determination is illustrated in Figure 2.

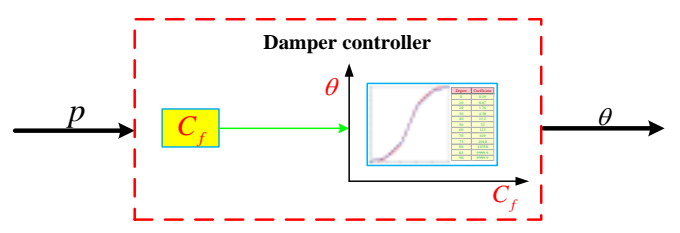

Fig. 2: Schematic diagram of damper position determination.

\section{Operating Steps of the Proposed Method}

Up to this moment, the proposed method has been completed. The procedure of determining the position using the proposed method is summarized as following steps:

Step 1: Calculating the posterior distribution of $\mathbf{w}$ using the training data $\mathrm{D}$, the $p(\mathbf{w} \mid \mathbf{P}, \alpha, \beta)$ needed for pressure prediction is obtained in this step;

Step 2: Selecting the appropriate value of the $\beta$, then the $p(\mathbf{P} \mid \mathbf{w}, \beta)$ needed for pressure prediction can be obtained;

Step 3: Calculating the optimal prediction of the pressure difference, i.e. outputs $\mathbf{P}=\left(p_{1}, p_{2}, \ldots, p_{k}\right)$ of the damper using given the desired airflow rate, i.e. inputs $\mathbf{q}=\left(q_{1}, q_{2}, \ldots, q_{i}\right)$;

Step 4: Calculating the friction coefficient $C_{f}$ of the damper according to the achieved pressure difference $\mathbf{P}$ in Step 3;

Step 5: Determining the damper position through a lookup table according to the obtained value of $C_{f}$ in Step 4.

The procedure can be well illustrated by a flowchart in Figure 3. 


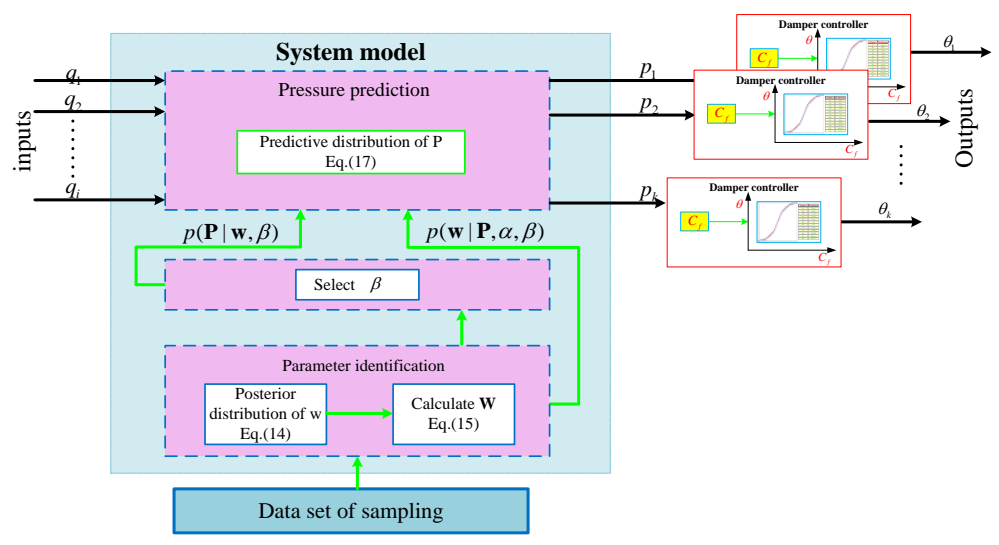

Figure 3: The procedure of the air balancing method.

\section{Performance Validation}

\subsection{Experiment platform}

In this study, an experimental rig together with the measurement instrumentation is developed to simulate a ventilation system. The ventilation system comprises two major parts: a fan and an air ducting system. The fan is a variable-speed centrifugal supply fan with backward curved blades, whose speed is controlled through direct DC voltage over the range from $0 \mathrm{~V}$ to $10 \mathrm{~V}$ provided from the controller. A PI controller was applied to control the fan speed using the measured duct static pressure (pressure sensor presented through blue dot in the figure) to keep the duct static pressure at specified setpoint. The major components in the air ducting system comprise three elbows, seven straight ducts, four tees and two transitions, five smart dampers $[15,16]$ and five terminals, which are the main components and extensively used in practice. The photo of the experimental rig is shown in Figure 4.

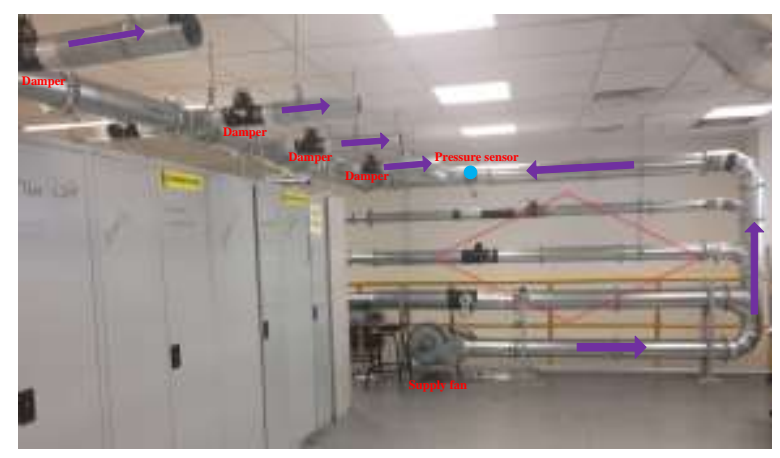

Fig. 4: Photo of the testbed having five terminals with smart damper.

\subsection{Relative error of airflow rate}

In this stage, the purpose is to validate the performance of modulating the position of the damper to achieve the desired airflow rate of the proposed method.

Firstly, the representative testing data set is chosen according to the characteristic curve of the damper. Four representative data sets of airflow rate, shown in Table 2, are chosen to be tested.

Secondly, the damper positions $\boldsymbol{\theta}=\left(\theta_{1}, \theta_{2}, \theta_{3}, \theta_{4}, \theta_{5}\right)$ of five terminals can be obtained using the developed model according to the selected desired airflow rate $\mathbf{q}=\left(q_{1}, q_{2}, q_{3}, q_{4}, q_{5}\right)$, and the positions of five dampers are modulated to the according to the angle $\boldsymbol{\theta}$.

Lastly, after the testbed settles down, the airflow rates $\hat{\mathbf{q}}=\left(\hat{q}_{1}, \hat{q}_{2}, \hat{q}_{3}, \hat{q}_{4}, \hat{q}_{5}\right)$ of five terminals are measured and captured. Then the error can be calculated through comparing $\mathbf{q}$ with $\hat{\mathbf{q}}$. Table 2 presented the results of air balancing in which the relative errors are all within $3.9 \%$. 
Table 2: Balancing result of five-terminal duct system.

\begin{tabular}{|c|c|c|c|c|c|c|}
\hline \multirow[t]{2}{*}{ No } & \multirow[t]{2}{*}{ Terminal } & Target & Desired & \multicolumn{2}{|l|}{ Tested } & \multirow[t]{2}{*}{ Relative } \\
\hline & & position & airflow & airflow rate & error & \\
\hline \multirow[t]{5}{*}{1} & 1 & 11.51 & 43 & 43.64 & & $1.5 \%$ \\
\hline & 2 & 14.10 & 35 & 35.12 & & $0.3 \%$ \\
\hline & 3 & 13.25 & 38 & 38.56 & & $1.5 \%$ \\
\hline & 4 & 1.35 & 26 & 25.71 & & $1.1 \%$ \\
\hline & 5 & 6.50 & 24 & 23.89 & & $0.5 \%$ \\
\hline \multirow[t]{5}{*}{2} & 1 & 35.40 & 21 & 21.64 & & $3.0 \%$ \\
\hline & 2 & 33.69 & 21 & 20.73 & & $1.3 \%$ \\
\hline & 3 & 40.23 & 17 & 16.83 & & $1.0 \%$ \\
\hline & 4 & 30.88 & 20 & 19.22 & & $3.9 \%$ \\
\hline & 5 & 40.69 & 13 & 13.49 & & $3.8 \%$ \\
\hline \multirow[t]{5}{*}{3} & 1 & 90 & 0 & 0 & & $0.0 \%$ \\
\hline & 2 & 90 & 0 & 0 & & $0.0 \%$ \\
\hline & 3 & 90 & 0 & 0 & & $0.0 \%$ \\
\hline & 4 & 90 & 0 & 0 & & $0.0 \%$ \\
\hline & 5 & 90 & 0 & 0 & & $0.0 \%$ \\
\hline \multirow[t]{5}{*}{4} & 1 & 10.59 & 40 & 40.58 & & $1.5 \%$ \\
\hline & 2 & 40.80 & 17 & 16.70 & & $1.8 \%$ \\
\hline & 3 & 51.17 & 10 & 9.64 & & $3.6 \%$ \\
\hline & 4 & 40.31 & 16 & 16.32 & & $2.0 \%$ \\
\hline & 5 & 90 & 0 & 0 & & $0.0 \%$ \\
\hline
\end{tabular}

\subsection{Energy saving potential of the proposed method}

This section shows the performance of the proposed method by trending a few measured variables, fan voltage, static pressure and airflow rate of terminal captured during the first 2-h data collection, providing useful information in regard to on-going energy savings and the reduction in static pressure compared the conventional system control.

Figure 5 presents the recorded measurements during the first 2-h experiment. The measurements were logged every $100 \mathrm{~ms}$ for the static pressure and fan input voltage, and every 2 minutes for airflow rate. Figure 5 (a) shows the measured static pressure at the fan outlet in which the performance of the PI controller can be seen. At the time of varying of the load, the pressure set-point was followed almost immediately. Figure 5 (c) shows the airflow rate of each terminal, which varied with the regulation of the damper position. Figure 5 (b) shows the input voltage to the fan from the controller during the test.

From Figure 5, it can be seen that the average voltage, used during the 2-h experiment is $3.9 \mathrm{~V}$, dropped on the average by $7.1 \%$ compared to the constant static pressure (the maximum of the voltage is $4.2 \mathrm{~V}$ during the first 2 -h experiment), which demonstrates that the proposed 5method saves energy compared to ACV system.

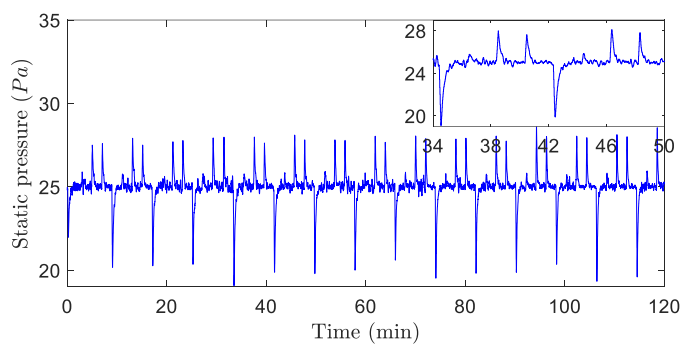

(a) Static pressure at the fan outlet. 


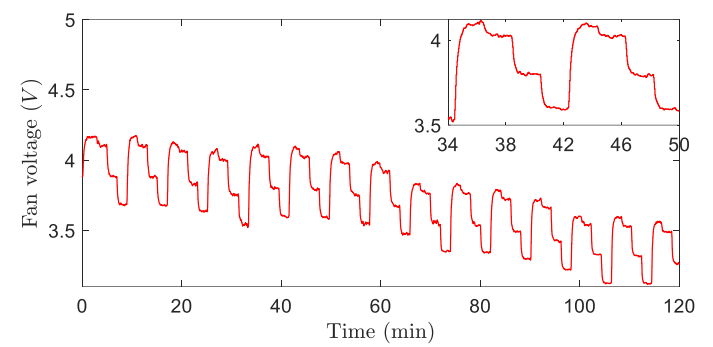

(b) Fan input voltage.

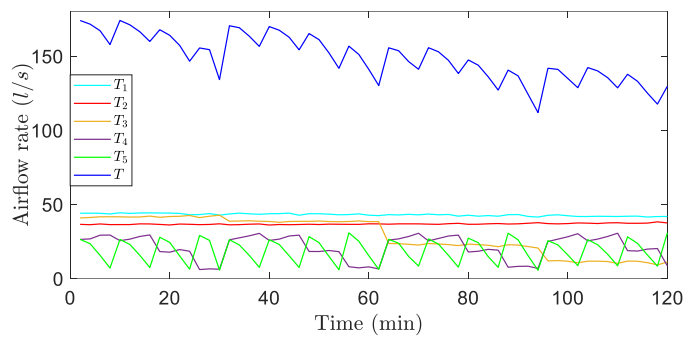

(c) Airflow rates of five terminal dampers (T1, T2, T3, T4, T5).

Fig. 5: Recorded measurements during a 2-h experiment.

\section{Conclusion}

This paper proposed an energy-saving oriented model-based air balancing method for the ventilation system, which can achieve the balancing among the terminals while at the same time minimize the energy use of the system. The method consisted of two layers: the first layer was a static pressure control scheme, the second one was an air balancing scheme. The first layer was used to maintain the static pressure of the main duct to its set-point to achieve the purpose of energy-saving, which was realized through the PI controller. The second layer was used to accurately balance the DCV system based on the measured airflow rate of each terminal by three steps. Firstly, the model of the system was build based on the component model and steady-state pressure balance. Secondly, the unknown parameters of the developed model are identified using the Bayesian linear regression machine learning algorithm. Lastly, the damper position was determined based on the empirical model of the damper to achieve the desired airflow rate.

The proposed method was validated in an experimental rig with five terminals. The evaluation of the method shows that this method is capable of balancing the DCV system given the desired airflow rates, and also has a large potential to save energy compared to the CAV and DCV system. The results show that the proposed method can achieve the desired airflow rates within $3.9 \%$ relative error, and the voltage of the fan can be reduced by $7.1 \%$ compare to CAV. system.

\section{Acknowledgements}

This work was partially funded by National Research Foundation of Singapore under the grant NRF2014EWTEIRP003-014, NRF2013EWT-EIRP004-019, NRF2011 NRF-CRP001-090, the scholarship from China Scholarship Council (CSC) (No. 201704000002) and the Science and technology plan project of Shandong higher education institutions (No. J16LN26, No. J17KA210).

\section{References}

[1] S. Rahnama, A. Afshari, N. C. Bergsøe, and S. Sadrizadeh, "Experimental study of the pressure reset con-trol strategy for energy-efficient fan operation: Part 1: Variable air volume ventilation system with dampers," Energy and Buildings, vol. 139, pp. 72-77, 2017.

[2] A. Afram and F. Janabi-Sharifi, "Gray-box modeling and validation of residential HVAC system for control system design," Applied Energy, vol. 137, pp. 134-150, 2015.

[3] M.-L. Maripuu, "Demand Controlled Ventilation (DCV) Systems in Commercial Buildings," Functional Requirements on Systems and Components (Chalmers University of Technology, Building Services Engineering), Gothenburg, 2009.

[4] "ANSI/ASHRAE standard 111-2008 (RA2017): Measurement, testing, adjusting and balancing of building HVAC systems," American Society of Heating, Refrigerating and Air-conditioning Engineers. 
[5] G. S. Okochi and Y. Yao, "A review of recent developments and technological advancements of variable-air-volume (VAV) air-conditioning systems," Renewable and Sustainable Energy Reviews, vol. 59, pp. 784-817, 2016.

[6] F. Pedranzini, L. P.M. Colombo, and C. Joppolo, "A non-iterative method for Testing, Adjusting and Bal-ancing (TAB) air ducts systems: Theory, practical procedure and validation," pp. 322-330, 2013.

[7] J. Tamminen, T. Ahonen, J. Ahola, and S. Hammo, "Fan pressure-based testing, adjusting, and balancing of a ventilation system," Energy Efficiency, vol. 9, pp. 425-433, 2016.

[8] S. Englander and L. Norford, Saving fan energy in VAV systems - part 2: supply fan control for static pressure minimization using DDC zone feedback," pp. 19-32, 1992.

[9] S. Rahnama, A. Afshari, N. C. Bergsøe, S. Sadrizadeh, and G. Hultmark, "Experimental study of the pres-sure reset control strategy for energy-efficient fan operation - Part 2: Variable air volume ventilation sys-tem with decentralized fans," Energy and Buildings, vol. 172, pp. 249-256, 2018.

[10] G. Jing, W. Cai, D. Zhai, S. Liu, and C. Cui, "A model-based air balancing method of a ventilation sys-tem," Energy and Buildings, vol. 174, pp. 506-512, 2018.

[11] G. Jing, W. Cai, H. Chen, D. Zhai, C. Cui, and X. Yin, "An air balancing method using support vector ma-chine for a ventilation system," Building and Environment, pp. 487-495, 2018.

[12] Ashrae Duct Fitting Database: Version 5.0, 2008.

[13] T. Walter and M. D. Sohn, "A regression-based approach to estimating retrofit savings using the Building Performance Database," Applied energy, vol. 179, pp. 996-1005, 2016.

[14] S. Wang, X. Sun, and U. Lall, "A hierarchical Bayesian regression model for predicting summer residential electricity demand across the USA," Energy, vol. 140, pp. 601-611, 2017.

[15] C. Cui, W. Cai, and H. Chen, "Airflow measurements using averaging Pitot tube under restricted conditions," Building and Environment, vol. 139, pp. 17-26, 2018.

[16] C. Cui, X. Zhang, W. Cai, and G. Jing, "A novel online air balancing method for the ventilation duct system via distributed cooperative control," Building and Environment, vol. 146, pp. 177-189, 2018. 